\title{
Hospitalization of Mental Disorders in Saint Louis County: "Where You Live Matters"
}

\author{
Echo Wang* and Lara D. Dame \\ St. Louis Department of Public Health, Saint Louis, MO, USA
}

\section{Objective}

We used hospitalization rates for mental disorders to determine utilization patterns and the need for community-based mental health services.

\section{Introduction}

Hospitalization rates for mental health disorders provide important information to help us prioritize community needs for mental health and urgent care plantation. In Saint Louis County, there were over 13,000 hospitalizations for mental disorders between 2010 and 2014. For all age groups, depressive disorders, including major depression and mood disorder not-otherwise-specified, were the most common primary diagnostic grouping for hospitalizations among mental disorders, followed by bipolar disorder. In 2012, The Saint Louis County Department of Planning defined 5 geographic areas (Inner North, Outer North, South, West and Central) within and crossing Saint Louis County's borders. Among them, the Inner North has the greatest poverty, as opposed to the West which has the least. These geographic areas, along with neighborhood poverty level, were analyzed to better understand the demographics of Saint Louis County residents experiencing mental disorders.

\section{Methods}

Hospitalization for a mental disorder - that is, a principal diagnosis of International Classification of Disease, Ninth Revision, code 290 - 319 - among Saint Louis County residents from 2010 to 2014 were obtained from the Missouri Department of Health and Senior Services (DHSS), Bureau of Health Care Analysis and Data Dissemination. Hospitalization rate was calculated by age, gender/sex, race/ethnicity, neighborhood poverty and geographic area using SAS 9.4.

The five geographic areas were created by the Saint Louis County Department of Planning for the 2012 Citizen Survey, which were defined based on the 49 ZIP codes within and crossing Saint Louis County's borders. ESRI ArcGIS was used to assign each census tract to one of the five survey areas based on having greater than 50 percent of its area falling within a particular survey area.

The maps were created using ESRI ArcMap version 10.3. The maps compare geographic and social economic patterns of rates of hospitalization of a mental disorder in Saint Louis County by census tract.

\section{Results}

The greatest burden of mental health-related hospitalizations was among children ages 15-17, Black/African Americans, and neighborhoods with "high" poverty. Hospitalization rates of mental disorders among children (age 0 - 17) increased from 67.3 to 81.1 per 10,000 from 2010 to 2014; among adults (18+), rates increased from 124.7 to 134.8 per 10,000 from 2010 to 2014. From 2010 to 2014, children living in the Inner North area were more than twice as likely to be hospitalized for a mental disorder as children who were living in the West. Similarly, hospitalization rates for mental disorders among adults living in the Inner North area were nearly three times greater than adults living in the West.

\section{Conclusions}

As illustrated by the maps, there is an obvious, positive association between poverty level and mental health-related hospitalizations among residents of Saint Louis County. Thus, although a likely overlooked policy concern, heightened focus on community-based mental health care facilities in certain areas, specifically the Outer and Inner North regions, may be both ethical and cost-effective. Furthermore, early prevention should be developed and introduced to children at the transition age period (15-17).

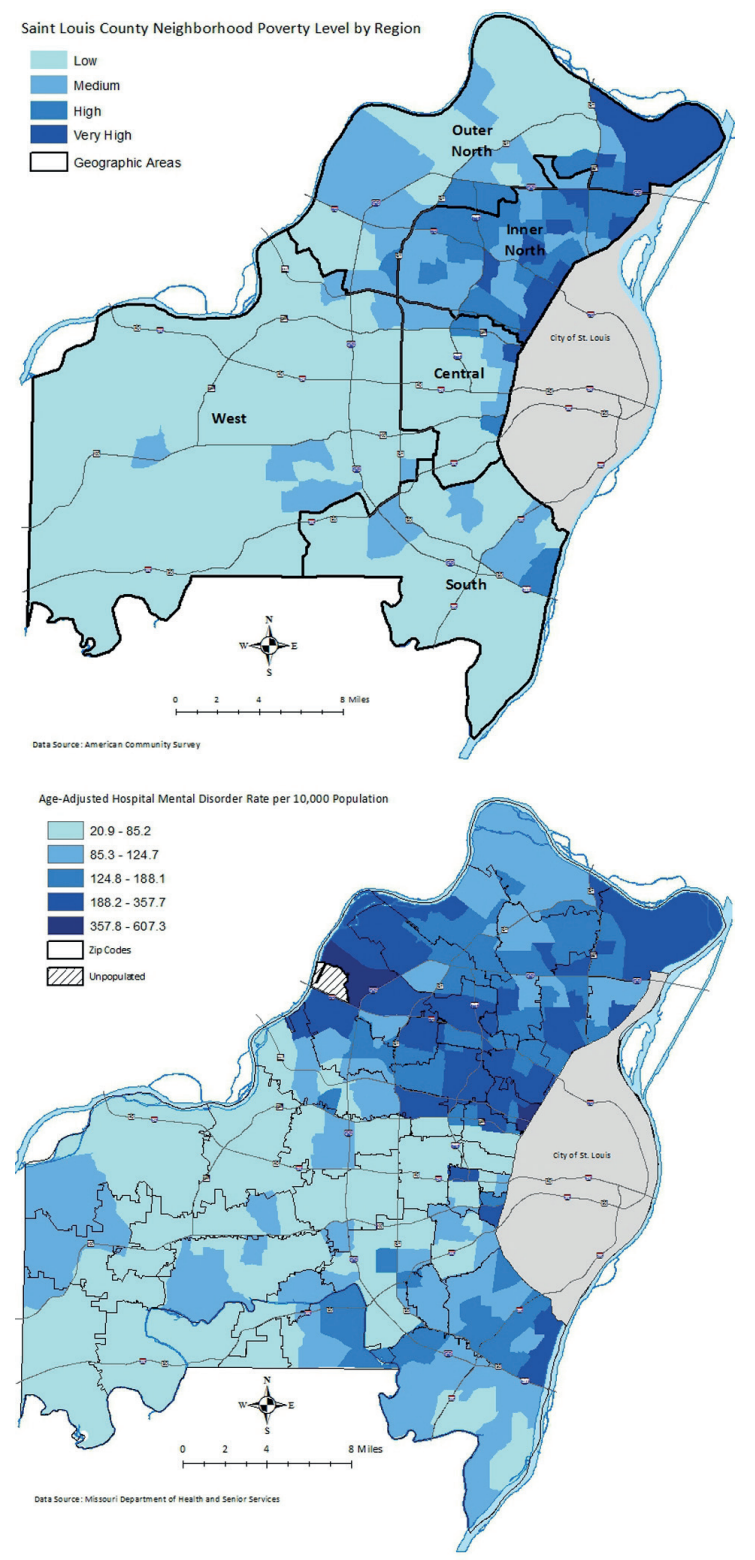




\section{ISDS 2018 Conference Abstracts}

Keywords

mental health; disparity; health equity; poverty; at risk

\section{Acknowledgments}

To Saint Louis Department of Public Health Chronic Disease Team

\section{References}

Wojas E, Meausoone V, Norman C. Adult Psychiatric Hospitalizations in New York City. Department of Health and Mental Hygiene: Epi Data Brief (71); June 2016.

\section{*Echo Wang}

E-mail: ewang@stlouisco.com 